\title{
A miniaturized germanium-doped silicon dioxide-based surface plasmon resonance waveguide sensor for immunoassay detection
}

\author{
Jhen-Gang Huang ${ }^{\mathrm{a}}$, Chen-Lung Lee ${ }^{\mathrm{a}}$, Hsueh-Min Lin ${ }^{\mathrm{b}}$, Tsung-Liang Chuang ${ }^{\mathrm{a}}$, \\ Way-Seen Wang ${ }^{\mathrm{d}}$, Rong-Huey Juang ${ }^{\mathrm{e}}$, Ching-Ho Wang ${ }^{\mathrm{f}}$, Chih Kung Lee ${ }^{\mathrm{g}}$, \\ Shi-Ming Lin ${ }^{\mathrm{h}}$, Chii-Wann Lin ${ }^{\mathrm{a}, \mathrm{b}, \mathrm{c}, *}$ \\ ${ }^{\text {a }}$ Institute of Biomedical Engineering, National Taiwan University, Taipei, Taiwan \\ ${ }^{\mathrm{b}}$ Department of Electrical Engineering, National Taiwan University, Taipei, Taiwan \\ ${ }^{\mathrm{c}}$ Center for Nano Science and Technology, National Taiwan University, Taipei, Taiwan \\ ${ }^{\mathrm{d}}$ Institute of Electro-Optical Engineering, National Taiwan University, Taipei, Taiwan \\ ${ }^{\mathrm{e}}$ Department of Biochemical Science and Technology, National Taiwan University, Taipei, Taiwan \\ ${ }^{\mathrm{f}}$ Department of Veterinary Medicine, National Taiwan University, Taipei, Taiwan \\ ${ }^{\mathrm{g}}$ Institute of Applied Mechanics, National Taiwan University, Taipei, Taiwan \\ ${ }^{\text {h }}$ Center for Optoelectronic Biomedicine, National Taiwan University, Taipei, Taiwan \\ Received 15 February 2006; received in revised form 22 July 2006; accepted 25 July 2006 \\ Available online 8 September 2006
}

\begin{abstract}
A surface plasmon resonance (SPR) waveguide immunosensor fabricated by germanium-doped silicon dioxide was investigated in this study. The designed waveguide sensor consisted of a $10 \mu \mathrm{m} \mathrm{SiO}{ }_{2}$ substrate layer $(n=1.469)$, a $10 \mu \mathrm{m} \mathrm{Ge-SiO}{ }_{2}$ channel guide $(n=1.492)$ and a $50 \mathrm{~nm}$ gold film layer for immobilization of biomolecules and SPR signal detection. The resultant spectral signal was measured by a portable spectrophotometer, where the sensor was aligned by a custom-designed micro-positioner. The results of the glycerol calibration standards showed that the resonance wavelength shifted from 628 to $758 \mathrm{~nm}$ due to changes of refractive index from 1.36 to 1.418 . Flow-through immunoassay on waveguide sensors also showed the interactions of protein A, monoclonal antibody (mAb ALV-J) and avian leucosis virus (ALVs) resulted in wavelength shifting of 4.17, 3.03 and $2.18 \mathrm{~nm}$, respectively. The SPR dynamic interaction could also be demonstrated successfully in 4 min as the sensor was integrated with a lateral flow nitrocellulose strip. These results suggest that SPR detection could be carried out on designed waveguide sensor, and the integration of nitrocellulose strip for sample filtering and fluid carrier would facilitate applications in point-of-care portable system.
\end{abstract}

(C) 2006 Elsevier B.V. All rights reserved.

Keywords: Surface plasmon resonance; Waveguide; Immunosensor

\section{Introduction}

Surface plasmon resonance (SPR) is an optical phenomenon, which is highly interfered by the refractive index of dielectric material on the metal layer on which surface plasmon wave (SPW) existed. Since SPR has the advantages of non-labeling, suitable for real time detection and high sensitivity, it had been extensively used for biomedical researches in the past decade (Chien and Chen, 2004; Salamon et al., 1997; Hug et al., 2001).

\footnotetext{
* Corresponding author at: Institute of Biomedical Engineering, National Taiwan University, No. 1, Sec. 4, Roosevelt Road, Taipei 106, Taiwan, ROC. Tel.: +886 233665271 ; fax: +886223620586

E-mail address: cwlinx@ntu.edu.tw (C.-W. Lin).
}

Traditionally, SPR detection utilized Otto or Kretschmann strictures, which used the prism coupling method to excite SPR on the surface, were the major platforms for SPR sensing (Kretschmann and Raether, 1968; Otto, 1968). However, the large size of the prism and the mechanical equipment required for angular modulation on SPR sensors restricted its development in miniaturized systems. If the coupling method is changed from prism to integrated optics devices such as fiber optics, the sensor size could be easily miniaturized. Moreover, if angular modulation was replaced by wavelength modulation, the spectrometer could reduce the detection time and detection system cost while simultaneously improving portability. There have been several reports of SPR optical sensors based on integrated optics structure (Dostálek et al., 2001; Hug et al., 2001; Liron et al., 2002; 
Suzuki et al., 2005; Zourob et al., 2005). Most of these sensors were fabricated as a slab waveguide structure, which could be used for the detection of average changes on the surface of dielectric materials. The other sensor developed by Slavík et al. $(1999,2001)$ used a fiber optics SPR sensor, but the fabrication of polished single mode fiber optics might not be suitable for possible mass production. In this study, a SPR waveguide sensor fabricated by germanium-doped silicon dioxide was investigated by using semiconductor compatible process of plasma enhanced chemical vapor deposition (PECVD). This would allow precise control of dopant concentration for the refractive index difference between core and cladding layer and thus fine tuning of the plasmon resonance wavelength. The integration with a flowthrough fluidic system or a nitrocellulose membrane would allow real time detection of SPR changes due to biomolecular interactions. In addition, the silica-based device can be integrated with photo detector and light source for parallel detection in the future.

\section{Principle}

The interface between metal and dielectric materials may support charge density oscillations, which are called surface plasmons. These occur at a different frequency than the bulk oscillations and are confined to the surface and exponentially decay on both sides of the interface. The coupling condition for the surface plasmon resonance to occur is

$k_{\mathrm{sp}}=\frac{\omega}{c} \sqrt{\frac{\varepsilon_{1} \varepsilon_{2}}{\varepsilon_{1}+\varepsilon_{2}}}$,

where $k_{\mathrm{sp}}, \omega, \varepsilon_{1}$ and $\varepsilon_{2}$ is the wave number of the surface plasmon wave, the frequency of the electric field along the surface plane, and the dielectric constants of the metal and dielectric layer, respectively.

From the above equation, when the dielectric constant of material on the metal layer is alternated, it could be detected by the variation of the resonance angle of the incident wave or the resonance wavelength. In this research, a waveguide with gold sensing film was used as the SPR sensor. As a sample was applied on the gold films, we would measure the shifting of the resonance wavelength and its intensity changes by a spectrophotometer.

\section{Materials and methods}

\subsection{Chemical}

Unless otherwise described, all reagents used in the research were reagent grade and prepared with Milli-Q deionized water. Biological reagent included antibodies and antigens were prepared in $\mathrm{pH} 7.2$ phosphate buffer solution (PBS). Glycerol, 1-ethyl-3-(3-dimethylaminopropyl) carbodimide (EDC), $\mathrm{N}$-hydroxysuccinimide (NHS) and protein A were obtained from Sigma. Linker, which consisted of octal-alkanethiol with carboxyl functional groups, was synthesized in laboratory.
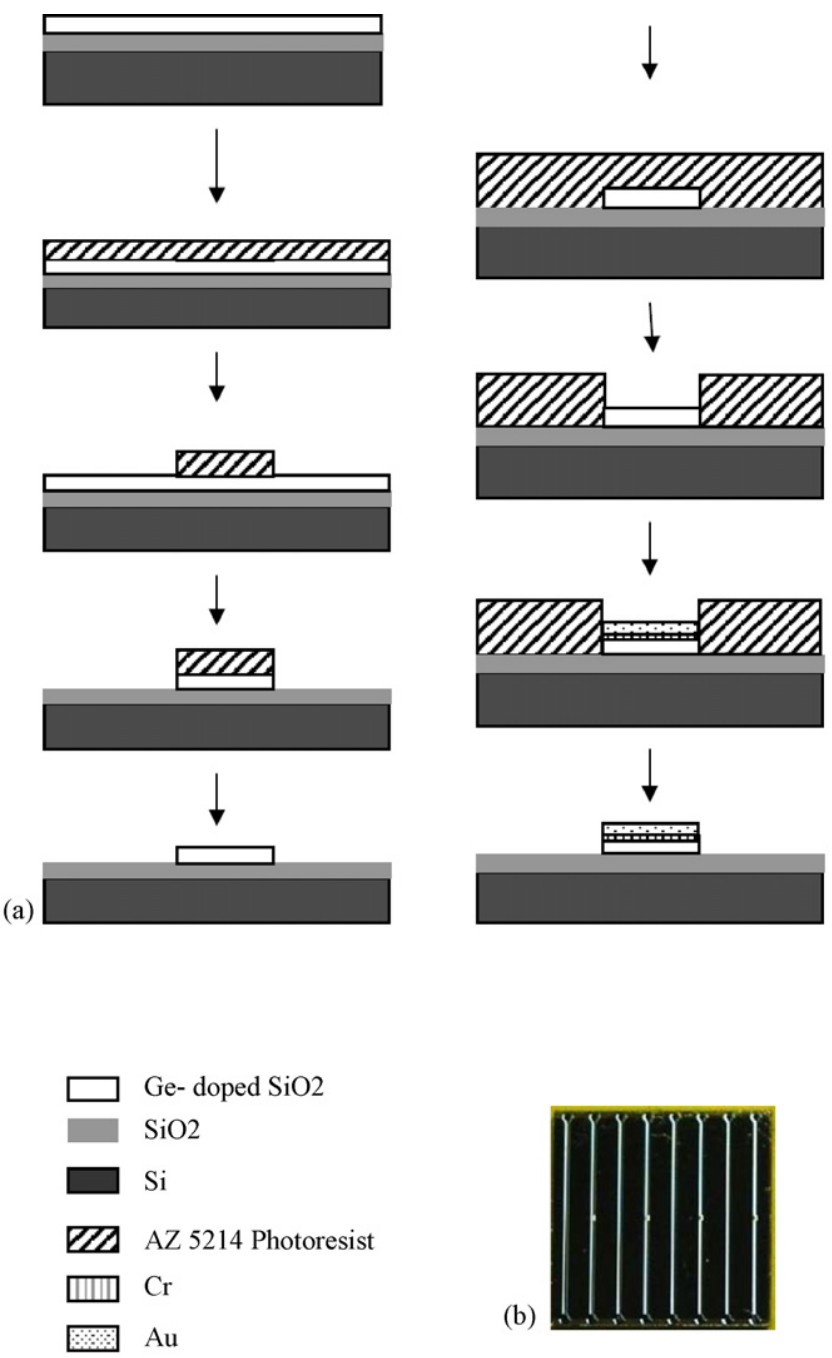

(b)

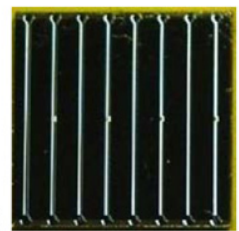

Fig. 1. (a) Fabrication process of waveguide immunosensor and (b) photograph of waveguide sensor. The gold spot on the waveguide was the SPR sensing area.

\subsection{Design and fabrication of waveguide sensor}

To optimize the electromagnetic field profile within the waveguide structure, a simulation based on various layer thickness of waveguide was accomplished by BeamPROP (Version 2.1, RSoft Design Group, Inc.). Waveguide sensors were then fabricated according to the process flow shown in Fig. 1a. First, a silicon wafer was washed with piranha solution $\left(\mathrm{H}_{2} \mathrm{SO}_{4}: \mathrm{H}_{2} \mathrm{O}_{2}=3: 1\right)$ at $80^{\circ} \mathrm{C}$ for $5 \mathrm{~min}$ and $1: 3$ diluted buffered oxide etchent (BOE) for $30 \mathrm{~s}$. Then, silicon dioxide $(n=1.469)$ and germanium-doped silicon dioxide $(n=1.491)$ were deposited on the silicon wafer by plasma enhanced chemical vapor deposition (PECVD) at a thickness of $10 \mu \mathrm{m}$ each. To define the waveguide pattern on the Ge-doped $\mathrm{SiO}_{2}$, waveguide pattern was first defined onto the substrate by lithography, and then the substrate was etched by BOE. Finally, to fabricate the SPR detection area on the waveguide, another lithography process was applied to the substrate and evaporated with $1 \mathrm{~nm}$ $\mathrm{Cr}$ and $10 \mathrm{~nm}$ Au subsequently. After lift-off process and chip dicing the fabrication of waveguide chip was accomplished. The fabricated waveguide chip was shown in Fig. 1b. The dimensions 

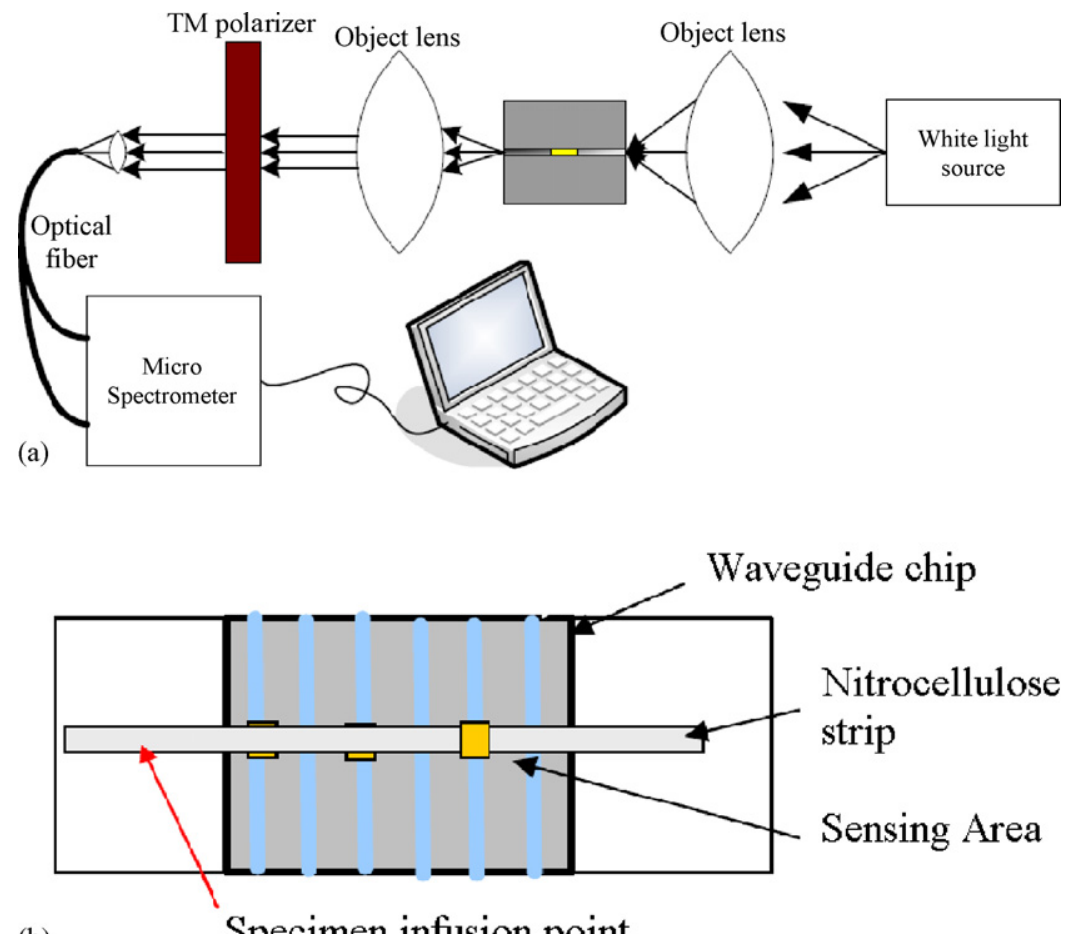

(b)

Specimen infusion point

Fig. 2. (a) Schematic representation of the SPR waveguide immunosensor measurement system and (b) schematic representation of the nitrocellulose strip integrated with the waveguide chip.

of the waveguide were $\sim 1 \mathrm{~cm}$ length, $200 \mu \mathrm{m}$ width. For the purpose of improving the light coupling efficiency into waveguide, a curvature structure at the end of the waveguide was designed and fabricated to facilitate the focus of incident light to waveguide.

\subsection{System set-up}

The detection system was shown as Fig. 2a. Light incident in and out of the waveguide was coupled by a pair of object lenses, which was mounted on a precision movement platform (Newport Co.) for alignment and focusing adjustment between the optical element and the waveguide. To collect the transmagnetic (TM) polarized light for SPR signal recognition, a polarizer was put between the object lens at the output end of the waveguide and the optical fiber used for signal collection. The SPR signal was then analyzed by a portable spectrometer (SD2000, Ocean Optics) connected to the optical fiber. Reagents for immunoassay were subsequently infused through the waveguide immunosensor by a homemade flow device. To develop a portable diagnostic system based on the waveguide immunosensor in this research, a nitrocellulose membrane was placed across the gold films of waveguide, as shown in Fig. $2 b$.

\subsection{Immunoassay}

To evaluate the detection efficiency of the waveguide immunosensor in clinical diagnosis, the operation process for avian leucosis virus (ALV) by flow-through immunoassay (FIA) was demonstrated on the waveguide immunosensor. A serum specimen with ALV particles and monoclonal antibodies (ALV$\mathrm{J} \mathrm{mAb}$ ) for ALV was obtained and purified in the laboratory. To immobilize the $\mathrm{mAb}$ on the gold sensing film, linker was first infused onto the gold film, and then carboxyl group of linker was activated by $400 \mathrm{mM}$ EDC and $100 \mathrm{mM}$ NHS. To improve the mAb immobilized orientation on the gold film, protein $\mathrm{A}$, which captures the $\mathrm{Fc}$ fragments of $\mathrm{mAb}$, was immobilized on the linker with the concentration of $0.1 \mathrm{mg} / \mathrm{ml}$ and captured mAb $(6 \mu \mathrm{g} / \mathrm{ml})$. Finally, serum with ALV particles was infused onto the gold film. The SPR signal was recorded as the resonance wavelength shifted throughout the process. To eliminate interference caused by non-specific adsorption of impurity, excess reagent was washed by PBS before another reagent was added.

\subsection{Develop the prototype of portable system}

As nitrocellulose membrane, could transport fluid and filter impurity in biological specimens at the same time, it was used to simplify the operation processes of immunoassay in the portable system. First, mAb for the ALV was immobilized on the sensing area, and then the nitrocellulose strip was fixed across the sensing area of the waveguide sensor. After the serum with ALV particles was added on one side of the nitrocellulose strip, PBS was dropped on the nitrocellulose strip. This process was used to elute the ALV specimens toward the sensing area $\sim 1 \mathrm{~cm}$ away from sample application point. SPR signal was monitored by the intensity variation at $570 \mathrm{~nm}$ from the time the ALV sample was applied to $10 \mathrm{~min}$ afterward. 

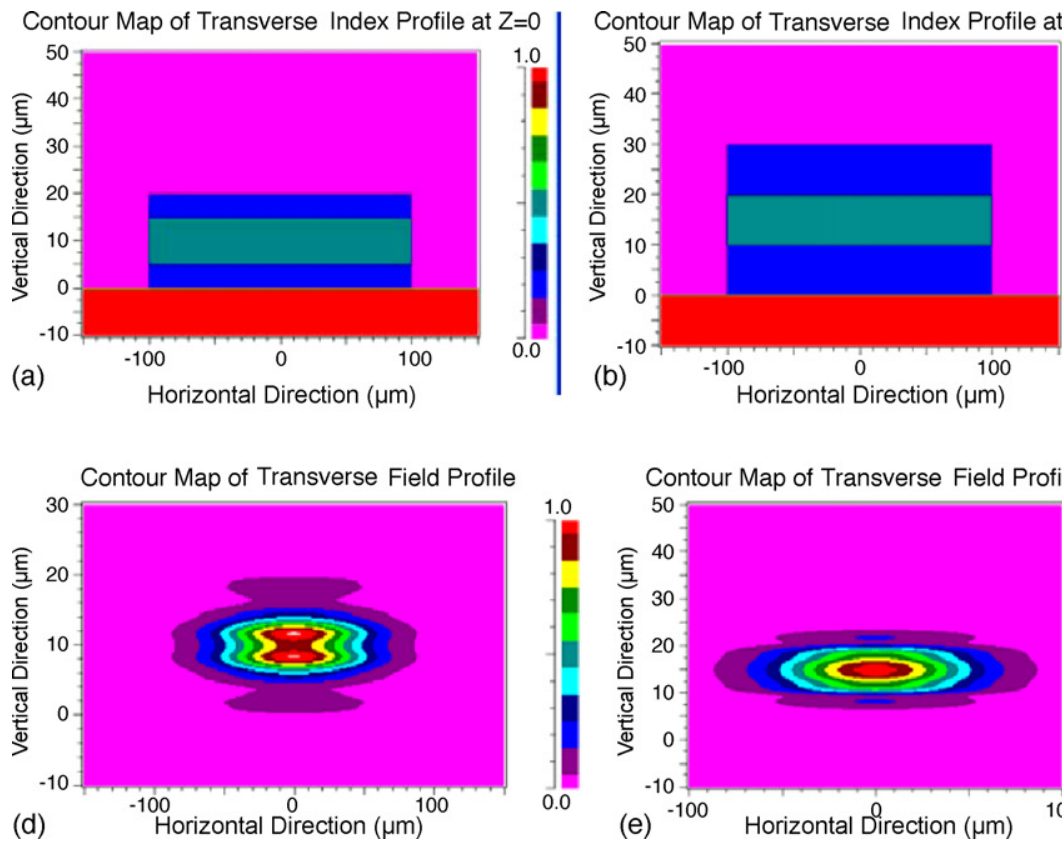

(e) ${ }^{-100}$ Horizontal Direction $(\mu \mathrm{m})$
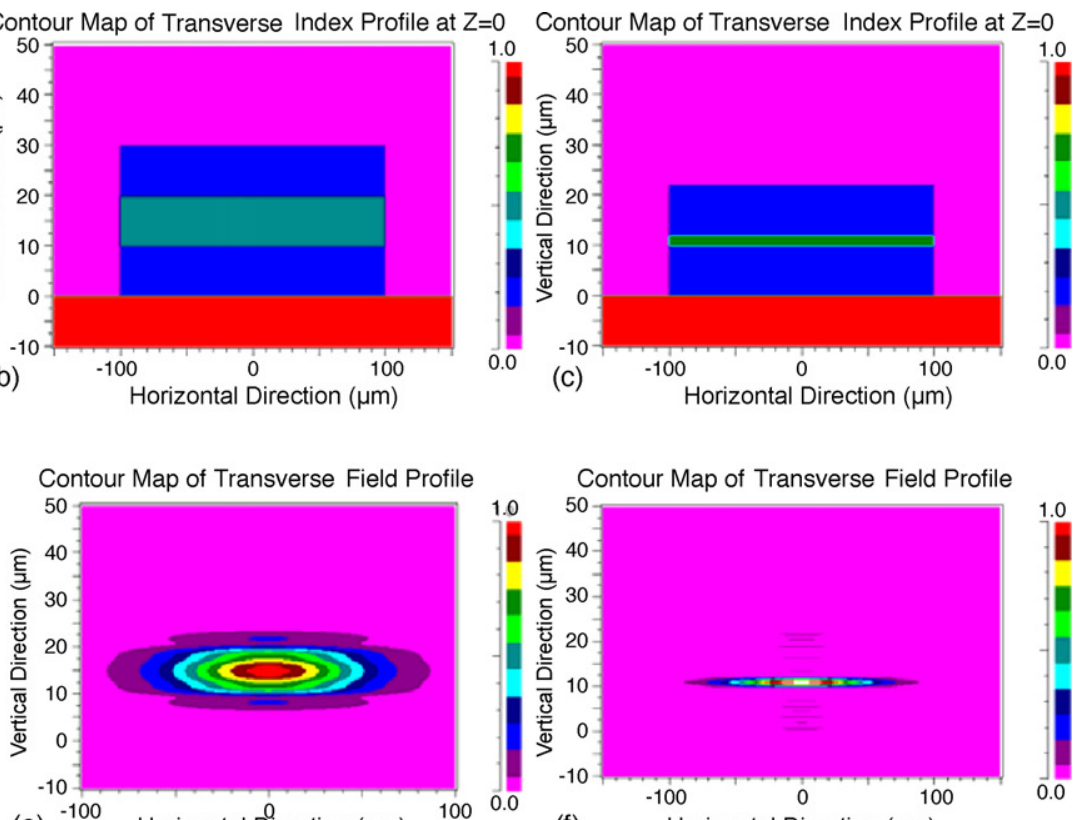

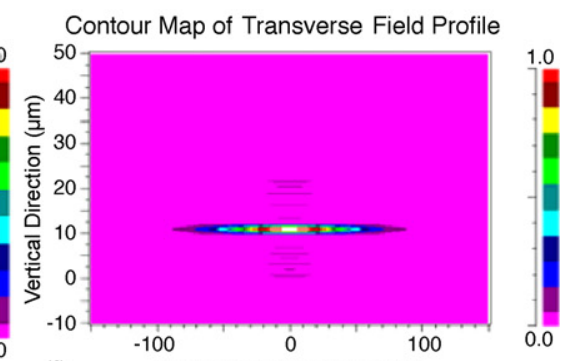

(f) Horizontal Direction $(\mu \mathrm{m})$

Fig. 3. Simulation structures and results of waveguide structure: (a) waveguide structure of $10 \mu \mathrm{m}$ core and $5 \mu \mathrm{m}$ cladding; (b) waveguide structure of $10 \mu \mathrm{m}$ core and $10 \mu \mathrm{m}$ cladding; (c) waveguide structure of $1.8 \mu \mathrm{m}$ core and $10 \mu \mathrm{m}$ cladding; (d) contour map of optical field in waveguide structure (a); (e) contour map of optical field in waveguide structure (b); (f) contour map of optical field in waveguide structure (c).

\section{Results}

\subsection{Simulations of waveguide structure}

The optimal electromagnetic field profile within the waveguide structure can be calculated by using simulation program. The refractive index of the core and cladding layers was 1.491 and 1.469 , respectively. Fig. $3 \mathrm{~d}$ and e shows that when the thickness of the core layer was $10 \mu \mathrm{m}$, the thickness of the cladding layer should at least $10 \mu \mathrm{m}$ to confine the optical field in the core structure as light was transmitted through the waveguide. Fig. 3d and $\mathrm{f}$ also shows that when the thickness of the cladding layer was $10 \mu \mathrm{m}$, a core thickness of 10 and $1.8 \mu \mathrm{m}$ would both confine well within the waveguide. As a thicker core would confine more light within the waveguide and create higher sensitivity. Based on considerations to improve sensitivity and reduce fabrication cost, thickness of $10 \mu \mathrm{m}$ for both core and cladding was used in this study.

\subsection{Influence of fabrication process to waveguide sensor}

Since sensitivity of SPR detection was highly related to the profile of the waveguide, such as the roughness and cross-section shape of the waveguide. The waveguide pattern fabricated was analyzed by a surface profiler and optical microscopy after fabrication. If the waveguide washed by 1:4 diluted BOE, photoresist would be peeled off after $1 \mathrm{~h}$ and thus thickness of waveguide was only $3 \mu \mathrm{m}$. As the thickness of photoresist or hard bake time was increased, a waveguide of nearly $10 \mu \mathrm{m}$ thick would be fabricated successfully. However, the cross-section of waveguide showed $70 \mu \mathrm{m}$ width at the top and $200 \mu \mathrm{m}$ width at the bottom. It was proposed that the trapezoid shape of the waveguide might be caused by the undercut of $\mathrm{BOE}$ to $\mathrm{SiO}_{2}$ under photoresist. The waveguide showed a smooth sidewall suitable for optical application. It was concluded that as the concentration of BOE was decreased, the etching rate of BOE would also be decreased, which could decreased the roughness of waveguide.

\subsection{Influence of waveguide structure to spectral characteristics}

The effect of the length of sensing area on the SPR signal was measured by three different lengths of gold sensing films, 200, 250 and $500 \mu \mathrm{m}$ and then tested by $20 \%$ glycerol solution with refractive index of 1.36. The results were shown in Fig. 4. It was

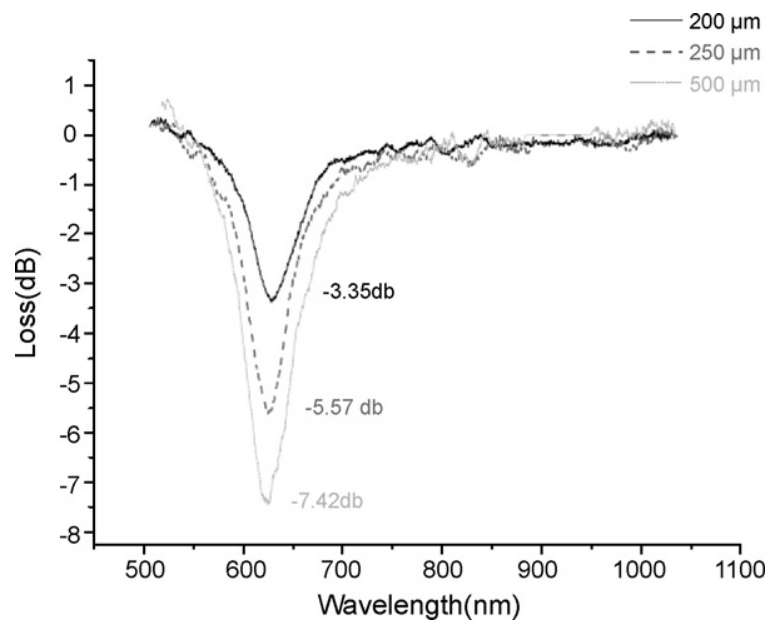

Fig. 4. Power loss response of waveguide to glycerol solution with various sensing area lengths $(n=1.360)$ 
found that the attenuation of transmitted light, i.e., power loss in $\mathrm{dB}$, decrease from 3.3 to $7.42 \mathrm{~dB}$ as the length of the sensing area was increased from 200 to $500 \mu \mathrm{m}$. With the enhancement in SPR response, we can expect the increment in sensitivity of fabricated devices. However, it is a trade-off between sample volume and sensor response.

\subsection{Response of waveguide immunosensor}

To evaluate the SPR response of the waveguide sensor to samples with different refractive indices, glycerol solutions with various concentrations were used as standard calibration samples. The results were shown in Fig. 5a. It could be found as the refractive index of glycerol was increased from 1.36 to 1.418 , the resonance wavelength of the SPR signal also increased from 628 to $758 \mathrm{~nm}$. The calculated sensitivity of the waveguide sensor was about $4 \times 10^{-4} \mathrm{RI} / \mathrm{nm}$. The comparison of the regression curves of experimental data and simulated results gives very small discrepancy, which is attributed to the surface roughness or structure defect of waveguide. Errors between simulated and experimental results were found less than $1.8 \%$ for all results.

A complete process of immunoassay could be accomplished by our simple flow-through apparatus for specimens with ALV and $\mathrm{mAb}$. SPR signal for biomolecular interactions could be monitored by the system set-up and the result was shown in Fig. 5b. It was found the wavelength response of linker solution $(n=1.332)$ was $593 \mathrm{~nm}$, which was approximate the simulated
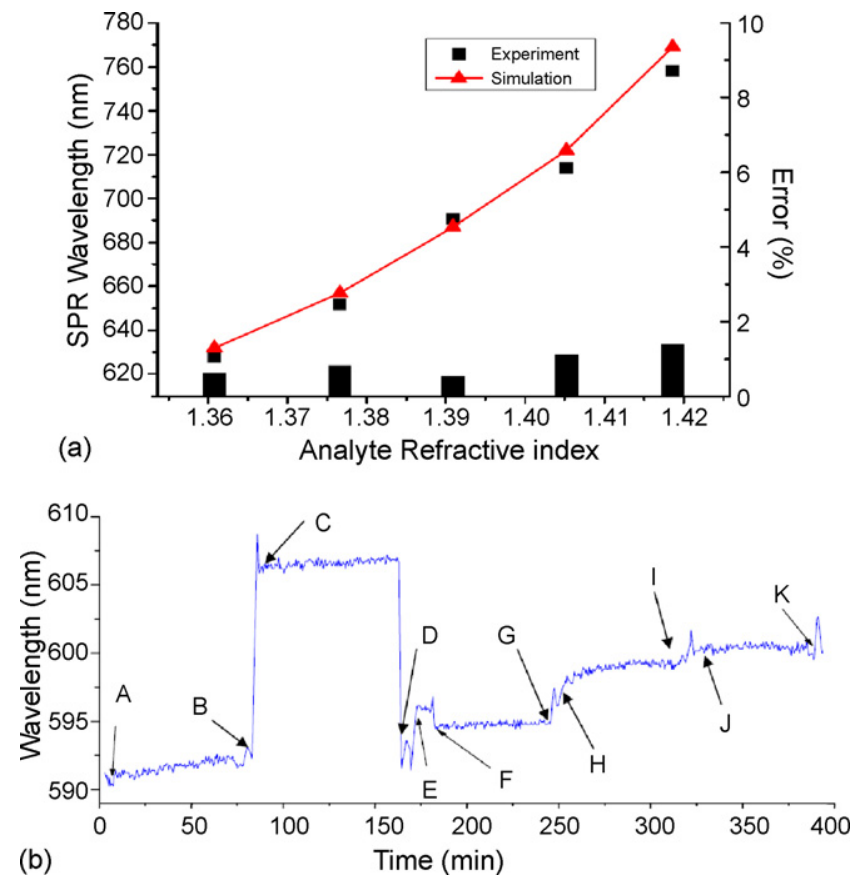

Fig. 5. (a) Comparison of the wavelength response of experimental (square) and simulation (triangle) results, percentage errors between the two results were showed as bar chart to axis at right-hand and (b) flow-through immunoassay of mAb-ALV interaction. Letters indicate reagent added: (A) linker; (C) EDC/NHS; (E) protein A; (F) diluted protein A; (H) mAb; (J) ALV; (B), (D), $(\mathrm{F}),(\mathrm{G}),(\mathrm{I})$ and $(\mathrm{K})$ was PBS wash.
Table 1

Resonance wavelength shifted in FIA

\begin{tabular}{llcl}
\hline Reagent & $\begin{array}{l}\text { Concentration } \\
(\mathrm{mg} / \mathrm{ml})\end{array}$ & $\begin{array}{l}\text { Molecular } \\
\text { size }(\mathrm{nm})\end{array}$ & $\begin{array}{l}\text { Wavelength } \\
\text { shifted }(\mathrm{nm})\end{array}$ \\
\hline EDC/NHS & $62.08 / 11.51$ & $<1$ & 0.6 \\
Protein A & 0.1 & $\sim 10$ & 4.17 \\
mAb & $6 \times 10^{-3}$ & $\sim 10$ & 3.03 \\
Antigen & Unknown & $\sim 120$ & 2.18
\end{tabular}

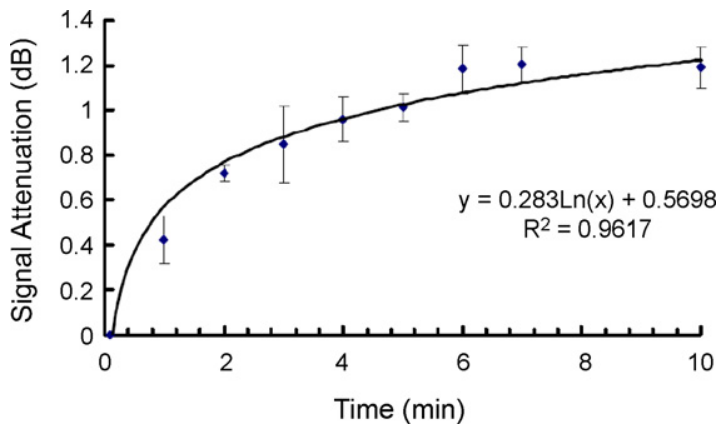

Fig. 6. Intensity variation of nitrocellulose integrated waveguide chip.

results (about $594 \mathrm{~nm}$ ) in Fig. 5a, the similar result was found for EDS/NHS ( $n=1.340$, response wavelength was $593.6 \mathrm{~nm}$ as simulated results was $595.6 \mathrm{~nm}$ ). So it was proposed the wavelength response in immunoassay also fulfilled the results of glycerol calibration standard. The resultant resonance wavelengths shifted of each reaction are shown in Table 1. It was found that the wavelength shift ranged from only $0.6 \mathrm{~nm}$ for small molecules such as EDC/NHS to $4.17 \mathrm{~nm}$ for large biomolecules such as protein A. Such a shift in SPR signal might be due to the differences in samples' molecular size, surface density and thus the effective refractive index.

\subsection{Simplified sample transportation via nitrocellulose strip}

The potential application for waveguide sensors was pointof-care portable diagnostic system. A waveguide immunosensor integrated with a nitrocellulose strip could serve as a prototype for portable applications. The intensity variation monitored at resonance wavelength of $570 \mathrm{~nm}$ was shown in Fig. 6. It was found that the power loss increased significantly in 2 min after ALV was dropped onto the sample loading area of nitrocellulose membrane. It is attributed to the increasing interactions of ALV to immobilized $\mathrm{mAb}$ on the sensing area during this period of time. However, the output signal approaching $95 \%$ of steady state value in about $4 \mathrm{~min}$ after ALV was infused through the nitrocellulose strip.

\section{Discussion}

In this paper, we reported the design of a Ge-doped Si-based SPR waveguide sensor for immunoassay. It has been fabricated by using PECVD processes for better control of core layer refractive index and thus the resonance wavelength in the visible 
light region. This can be calculated by using theoretical model of wave propagation in the dielectric waveguide and dispersion relationship for SPR coupling. If we assume the targeted biomolecules with refractive index in the range of 1.33-1.45, then the use of Ge-doped $\mathrm{SiO}_{2}(n=1.491)$ as core layer and $\mathrm{SiO}_{2}$ $(n=1.469)$ as cladding layer will result in two SPR resonance wavelengths, one at $499 \mathrm{~nm}$ and the other one at $643 \mathrm{~nm}$. However, the first peak at $499 \mathrm{~nm}$ shows very small responses to the refractive index changes. Thus, our measurements of absorption spectra are focusing around $643 \mathrm{~nm}$ red light regions. Our designed structure (200 $\mu \mathrm{m}$ width and $10 \mu \mathrm{m}$ height) will allow multimode of electromagnetic wave propagation within the waveguide. It is rather critical to have at least $10 \mu \mathrm{m}$ cladding layer in order to confine most of the EM energy within the core area.

The refractive index of the fabricated waveguide sensors is sensitive to the PECVD processing control of reactive gases flow volume, RF power, substrate temperature, vacuum pressure, and annealing temperature. We can achieve refractive index changes from 1.468 to 1.512 by doping $0-30 \%$ tri-methyl-germanium (TMG) gas in the deposition process. While the density or the lattice structure of the deposed Ge-doped silica is affected by above-mentioned processing parameters, we found that annealing process is critical for the final optical properties and etching rate of waveguide structure. Judging by the surface roughness of fabricated waveguide devices, the optimal annealing temperature is around $650{ }^{\circ} \mathrm{C}$ in our case. Our fabrication method is suitable for the bioanalytical applications due to the following reasons. First, refractive index of waveguide core layer can be adjusted by doping with TMG, which is safer than the diffusion of fluoride or other reactive gases such as, tri-methyl-gallium, arsine and ammonia. It is also cheaper than other fabrication technology such as ion exchanges or diffusion of $\mathrm{Yb}$ or Er. Finally, the application range of germanium-doped waveguide is in the visible light region, which is more suitable for bioanalytical detection by SPR than traditional waveguide fabricated by other technology, which was used for near infrared region for about 1300-1500 nm (Okamoto, 2000).

Our fabricated sensor could detected samples with refractive index from 1.36 to 1.418 with sensitivity of $4 \times 10^{-4} \mathrm{RI} / \mathrm{nm}$, which was slightly higher than waveguide sensor fabricated by ion exchanged method, which is about $4.8 \times 10^{-4} \mathrm{RI} / \mathrm{nm}$ in their report (Dostálek et al., 2001). The comparisons between theoretical calculation and experimental results of various calibration standards shows very good correlation and the variations are less than $1.8 \%$. The minor differences are attributed to the used optical constants in simulation might be different in Au ultra thin film of $50 \mathrm{~nm}$ thickness and the use of $1 \mathrm{~nm} \mathrm{Cr}$ as adhesive layer between $\mathrm{Au}$ and $\mathrm{Si}$.

From the time series measurement results shown in Fig. 5b, we can observe the dynamic responses of molecular interactions and compare wavelength difference due to individual molecules. The dynamics of alkanethiol self-assembly process takes time to develop on the surface and results in nearly no changes of SPR wavelength due to its small length $(\sim 1.4 \mathrm{~nm})$. The activation procedure of EDC results in $0.6 \mathrm{~nm}$ wavelength shift on top of bulk solution effect. The immobilization of protein A, cap- ture of mAb-J, and its interaction with ALVs shows 4.17, 3.03, and $2.18 \mathrm{~nm}$ changes, respectively in this measurement. It may be due to the differences in molecular size and packing density, which results in the lower effective refractive index. The use of lateral flow nitrocellulous strip for sample loading and filtering has been widely explored in the in vitro diagnostic devices (IVDs), recently. However, it is a novel idea to couple it with SPR waveguide sensor to provide possible quantitative readout. Our experimental result indicates that the intensity measurement at specific resonance wavelength $(570 \mathrm{~nm}$ in our case) can provide real time assay for influent samples. The flow velocity depends on the pore size of used NC membrane, and the particle size of target molecules. In our case, a $1 \mathrm{~cm}$ strip can almost reach $95 \%$ steady state in $4 \mathrm{~min}$.

\section{Conclusions}

In this study a SPR immunosensor based on a Ge-doped $\mathrm{SiO}_{2}$ waveguide was investigated. Response to refractive index and dynamic interaction of molecules observed by waveguide sensors investigated, and the simplified immunoassay carried out by nitrocellulose lateral flow strip was also be demonstrated. The results of the glycerol calibration standards and flow-through immunoassay on waveguide sensors showed the static and dynamic interactions changes of refractive index could be monitored by the waveguide SPR sensor. The successful delivery of biomolecules by a lateral flow nitrocellulose strip in 4 min also suggest that the integration of nitrocellulose strip for sample filtering and fluid carrier would facilitate applications in point-of-care portable system. In order to develop a portable total analysis system, we will try to integrate a planar light source and detector onto the waveguide sensor in the near future. Also a multiplex microfluidic system can be integrated onto the top layer of fabricated device to support the application of for high throughput system.

\section{Acknowledgements}

The authors are thankful for the financial support of the National Science and Technology Project of Biotechnology and Pharmaceuticals (NSTPBP) NSC94-2323-B002-013, -014 (National Science Council, Taiwan, ROC), and 94AS-5.1.3-S-a4 (Council of Agriculture, Taiwan, ROC).

\section{References}

Chien, F.C., Chen, S.J., 2004. Biosens. Bioelectr. 20, 633-642.

Dostálek, J., Čtyroký, J., Homola, J., Brynda, E., Skalský, M., Nekvindová, P., Špirková, J., Škvor, J., Schröfel, J., 2001. Sens. Actuators B 76, $8-12$.

Hug, T.S., Prenosil, J.E., Morbidelli, M., 2001. Biosens. Bioelectr. 16, 865 874.

Kretschmann, E., Raether, H., 1968. Z. Naturforsch. A 23, 2135-2136.

Liron, Z., Tender, L.M., Golden, J.P., Ligler, F.S., 2002. Biosens. Bioelectr. 17, 489-494.

Okamoto, K., 2000. Fundamentals of Optical Waveguides. 1st Academic Press, San Diego.

Otto, A., 1968. Z. Physik 216, 398-410. 
Salamon, Z., Macleod, H.A., Tollin, G., 1997. Biochim. Biophys. Acta 1331, $131-152$.

Slavík, R., Homola, J., Čtyroký, J., 1999. Sens. Actuators B 54, 74-79.

Slavík, R., Homola, J., Čtyroký, J., Brynda, E., 2001. Sens. Actuators B 74, 106-111.
Suzuki, A., Kondoh, J., Matsui, Y., Shiokawa, S., Suzuki, K., 2005. Sens. Actuators B 106, 383-387.

Zourob, M., Mohr, S., Brown, B.J.T., Fielden, P.R., McDonnell, M.B., Goddard, N.J., 2005. Biosens. Bioelectr. 21, 293-302. 